\title{
SMALL DEFORMATIONS OF A CLASS OF COMPACT NON-KÄHLER MANIFOLDS
}

\author{
L. ALESSANDRINI AND G. BASSANELLI \\ (Communicated by Clifford J. Earle, Jr.)
}

\begin{abstract}
Small deformations of Kähler manifolds are Kähler too; we prove here that this is not true for balanced manifolds nor, more generally, for $p$ Kähler manifolds $(p>1)$, i.e., the property of being $p$-Kähler is not stable under small deformations.
\end{abstract}

\section{INTRODUCTION}

The aim of this paper is to prove that if $M=\left\{M_{t}\right\}$ is a complex analytic family of compact complex manifolds, and $M_{t_{0}}$ is $p$-Kähler $(p>1)$, then, even if $\left|t-t_{0}\right|$ is small, $M_{t}$ may be a non $p$-Kähler manifold; i.e., the property of being $p$-Kähler is not stable under small deformations (see Theorem 2.3 for the definition of $p$-Kähler manifold).

It is well known that the situation is completely different in the case of Kähler manifolds [KS]. In what follows, we shall distinguish the case $p=\operatorname{dim} M_{t}-1:=$ $n-1$ (§4) from the general case (§3): indeed, an $(n-1)$-Kähler manifold is nothing but a manifold equipped with a balanced metric, i.e., a hermitian metric $h$ such that if $\omega$ is its Kähler form and $\delta$ is the formal adjoint of $d$ in the metric $h$, it holds that $\delta \omega=0$ (see [M] for a survey on balanced manifolds). The condition of being balanced (or co-Kähler) is, in a sense, dual to that of being Kähler, so it may be interesting to emphasize the different behaviour of small deformations of Kähler and balanced manifolds

\section{Preliminaries}

(See also [HK].) Suppose $V$ is a complex vector space of complex dimension $n$ and let $\sigma_{p}:=i^{p^{2}} 2^{-p}$.

Received by the editors June 20, 1989.

1980 Mathematics Subject Classification (1985 Revision). Primary 32G05.

This work is partially supported by MPI. The second author thanks the Department of Mathematics of the University of Trento for its kind hospitality during the preparation of this paper. 
2.1. Definition. (1) An element of $\Lambda^{p, 0}(V)$ that can be expressed as $v_{1} \wedge \cdots \wedge v_{p}$ with $v_{j} \in V$ is called decomposable.

(2) $\psi \in \Lambda^{p, p}(V)$ is called strongly positive if $\psi$ can be written as $\Sigma_{j} \sigma_{p} \zeta_{j} \wedge$ $\bar{\zeta}_{j}$, with $\zeta_{j} \in \Lambda^{p, 0}(V)$ decomposable

(3) $\Omega \in \Lambda^{p, p}(V)$ is called weakly positive if $\Omega \wedge \psi$ is a positive volume for every strongly positive $(n-p, n-p)$-vector.

Let $S P^{p}$ and $W P^{p}$ denote the cones of strongly positive and weakly positive $(p, p)$-vectors.

Let $M$ be a connected complex manifold of dimension $n$; we denote, as usual, by $\Omega^{p}$ the sheaf of germs of holomorphic $p$-forms and by $E^{p}$ the sheaf of germs of $C^{\infty} p$-forms.

Moreover, let $E^{p, p}(M)_{\mathbf{R}}$ denote the Fréchet space of real $(p, p)$-forms, while $E_{p, p}^{\prime}(M)_{\mathbf{R}}$ denotes its dual space of real currents of bidimension $(p, p)$.

2.2. Definition. $\Omega \in E^{p, p}(M)_{\mathbf{R}}$ is called transverse if $\forall x \in M, \Omega_{x}$ belongs to the interior of the cone $W P^{p}\left(T_{x}^{\prime} M^{*}\right) . T \in E_{p, p}^{\prime}(M)_{\mathbf{R}}$ is called positive if $T(\Omega) \geq 0$ for every transverse $(p, p)$-form $\Omega$.

2.3. Theorem. Let $M$ be a compact complex manifold of dimension $n$, and $p$ an integer, $1 \leq p \leq n$. The following assentions are equivalent:

(1) There exists on $M$ a closed transverse $(p, p)$-form;

(2) There are no nontrivial positive $(p, p)$-currents which are $(p, p)$-components of boundaries.

In this case, $M$ is called a p-Kähler manifold.

Proof. See [AA].

Remarks. (see $[\mathrm{AA}]$ and $[\mathrm{AB}])$.

(1) $M$ is 1 -Kähler if and only if $M$ is Kähler.

(2) $M$ is $(n-1)$-Kähler if and only if $M$ is balanced.

(3) If $M$ is Kähler, then it is $p$-Kähler for every $p \leq n$.

More about $p$-Kähler manifolds can be found in $[\mathrm{AA}]$ and $[\mathrm{AB}]$; we only recall here the definition of $\eta \beta_{5}$, a nontrivial 3-Kähler manifold that we shall use in the next section.

Let $G$ be the following subgroup of $G L(4, \mathrm{C})$ :

$$
G:=\left\{X \in G L(4, \mathbf{C}) / X=\left(\begin{array}{cccc}
1 & z_{1} & z_{3} & z_{5} \\
0 & 1 & 0 & z_{2} \\
0 & 0 & 1 & z_{4} \\
0 & 0 & 0 & 1
\end{array}\right)\right\}
$$

and let $\Gamma$ be the subgroup of $G$ consisting of matrices with entries in $\mathbf{Z}[\sqrt{-1}]$. $\Gamma$ is a discrete subgroup of $G$ and $G / \Gamma$ becomes a holomorphically parallelisable compact connected complex manifold of dimension 5 , called $\eta \beta_{5}$. We can 
identify the complex Lie group $G$ with $\left(\mathbf{C}^{5}, *\right)$, where $\left(z_{1}, \ldots, z_{5}\right) *\left(w_{1}, \ldots, w_{5}\right)=\left(z_{1}+w_{1}, \ldots, z_{4}+w_{4}, z_{5}+w_{5}+z_{1} w_{2}+z_{3} w_{4}\right)$, and we can choose a basis $\left\{\phi_{j}\right\}_{1 \leq j \leq 5}$ for $\Omega^{1}\left(\eta \beta_{5}\right)$ such that $d \phi_{j}=0$ for $j=1, \ldots, 4$ and $d \phi_{5}=-\phi_{1} \wedge \phi_{2}-\phi_{3} \wedge \phi_{4}$. Call $\left\{\theta_{j}\right\}$ the dual basis.

2.5. Proposition. $\eta \beta_{5}$ is not Kähler nor 2-Kähler, it is $p$-Kähler for $p=3,4,5$. Proof. See $[\mathrm{AB}]$.

The theory of small deformations can be found in [K] or [KM, Chapter 4].

\section{THE CASE $p<n-1$}

Take $M=\eta \beta_{5}$ and $\psi(t):=t \theta_{1} \bar{\phi}_{1}, t \in \mathbf{C}$.

$\forall t \in \mathbf{C}$, the vector $(0,1)$-form $\psi(t)$ satisfies the equation

$$
\bar{\partial} \psi(t)-\frac{1}{2}[\psi(t), \psi(t)]=0,
$$

and therefore, by Kuranishi's theorem [K], $\psi(t)$ defines for $|t|<\varepsilon$ a locally complete complex analytic family of deformations $\left\{M_{t}\right\}$ of $M$.

By solving

$$
\left\{\begin{array}{l}
\bar{\partial} \zeta_{j}-\psi(t) \zeta_{j}=0, \\
\zeta_{j}(0)=z_{j},
\end{array} \quad j=1, \ldots, 5\right.
$$

we get

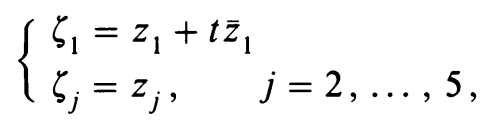

so that, if

$$
\phi_{j, t}=d \zeta_{j, t} \text { for } j=1, \ldots, 4
$$

and

$$
\phi_{5, t}=d \zeta_{5, t}+\frac{t \bar{\zeta}_{1, t}-\zeta_{1, t}}{1-|t|^{2}} d \zeta_{2, t}-\zeta_{3, t} d \zeta_{4, t}
$$

$\left\{\phi_{j, t}, \bar{\phi}_{j, t}\right\}_{j=1, \ldots, 5}$ becomes a basis for $H^{0}\left(M_{t}, E^{1}\right)$.

3.1. Proposition. For $|t|<\varepsilon, M_{t}$ is balanced, but it is not 3-Kähler if $t \neq 0$.

Proof. A 4-Kähler form for $M_{t}$ is $\Omega=\sigma_{4} \Sigma_{|A|=4}^{\prime} \phi_{A} \wedge \bar{\phi}_{A}$ (as usual, $\Sigma^{\prime}$ denotes the sum over all increasing multiindices, $A=\left(\alpha_{1}, \ldots, \alpha_{p}\right)$ and $\phi_{A}=\left(\phi_{\alpha_{1, t}} \wedge\right.$ $\left.\cdots \wedge \phi_{\alpha_{p, t}}\right)$.

Let $\eta$ be the $(2,2)$-component of $d\left(\phi_{1, t} \wedge \phi_{5, t} \wedge \bar{\phi}_{2, t}\right)$, i.e.,

$$
\eta=\frac{t}{1-|t|^{2}} \phi_{1, t} \wedge \phi_{2, t} \wedge \bar{\phi}_{1, t} \wedge \bar{\phi}_{2, t}
$$

and let $T_{\eta}$ be the real smooth $(3,3)$-current defined by $\eta$, that is,

$$
T_{\eta}(\alpha):=\int_{M} \eta \wedge \alpha
$$


Since, if $\alpha \in W P^{3}\left(M_{t}\right), \eta \wedge \alpha$ is a nonnegative multiple of the volume of $M_{t}$, $T_{\eta}$ is a positive $(3,3)$-current which is the $(3,3)$-component of a boundary and $T_{\eta}$ is trivial only for $t=0$. We can conclude by Theorem 2.3 .

\section{THE CASE $p=n-1$}

Let us choose the simplest example of a compact balanced non-Kähler manifold, the Iwasawa manifold $I_{3}$. Nakamura [N, pp. 94-96] builds up, via Kuranishi's theorem, a locally complete complex analytic family of deformations $M_{t}$ of $M_{0}=I_{3}$, depending on six effective parameters $t_{i j}$.

For our purpose it suffices to choose $t_{i j}=0$ for $(i, j) \neq(1,2)$. Call $t_{12}:=t$ : we get on $M_{t}$ coordinates $\left\{\zeta_{1, t}, \zeta_{2, t}, \zeta_{3, t}\right\}$ such that, if

$$
\phi_{j, t}=d \zeta_{j, t} \text { for } j=1,2 \text { and } \phi_{3, t}=d \zeta_{3, t}+\left(t \bar{\zeta}_{2, t}-\zeta_{1, t}\right) d \zeta_{2, t},
$$

then

$$
\left\{\phi_{j, t}, \bar{\phi}_{j, t}\right\}_{j=1,2,3} \text { becomes a basis for } H^{0}\left(M_{t}, E^{1}\right) \text {. }
$$

If $\Omega$ were a closed transverse $(2,2)$-form on $M_{t}$, by Stokes' theorem,

$$
0=\int_{M_{t}} d\left(\Omega \wedge \sigma_{1} \bar{t} \phi_{3, t}\right)=\int_{M_{t}} \Omega \wedge \sigma_{1} \bar{t} d \phi_{3, t}=-\int_{M_{t}} \Omega \wedge \sigma_{1}|t|^{2} \phi_{2, t} \wedge \bar{\phi}_{2, t},
$$

but, for $t \neq 0$, the last term is negative.

We have therefore proved the following:

4.1. Proposition. There exists a nonbalanced small deformation of $I_{3}$.

\section{REFERENCES}

[AA] L. Alessandrini and M. Andreatta, Closed transverse $(p, p)$-forms on compact complex manifolds, Compositio Math. 61 (1987), 181-200; Erratum, ibidem 63 (1987).

[AB] L. Allesandrini and G. Bassanelli, Compact p-Kähler manifolds (to appear).

[HK] R. Harvey and A. W. Knapp, Positive $(p, p)$-forms, Wirtinger's inequality and currents, in Proceedings Tulane Univ., Program on Value Distribution Theory in Complex Analysis and Related Topics in Differential Geometry 1972-73, Dekker, New York, 1974, 43-62.

[K] M. Kuranishi, On the locally complete family of complex analytic structures, Ann. Math. 75 (1962), 536-577.

[KM] K. Kodaira and J. Morrow, Complex Manifolds, Holt, Rinehart and Winston, New York, 1971.

[KS] K. Kodaira and D. C. Spencer, On deformations of complex analytic structures, III. Stability theorems for complex structures, Ann. Math. 71 (1960), 43-76.

[M] M. L. Michelson, On the existence of special metrics in complex geometry, Acta Math. 143 (1983), 261-295.

[N] I. Nakamura, Complex parallelisable manifolds and their small deformations, J. Differential Geom. 10 (1975), 85-112.

Dipartimento di Matematica, Università di Trento, I-38050 Povo (TN), Italy ITALY

Dipartimento di Matematica e Fisica, Università di Camerino, I-62032 Camerino (MC), 